\title{
Quantum Image Reversible Watermarking Scheme using a Combination of the most Significant Qubit and the Least Significant Qubit
}

\author{
Gaofeng Luo ${ }^{1}$, Ling Shi ${ }^{2, *}$, Tongcheng Huang ${ }^{1}$ and Zijuan Shi ${ }^{1,3}$ \\ ${ }^{1}$ College of Information Engineering, Shaoyang University, Shaoyang 422000, China \\ ${ }^{2}$ Department of Information Technology and Creativity, Shaoyang Polytechnic, Shaoyang 422000, China \\ ${ }^{3}$ Faculty of Education, University of Malaya, Kuala Lumpur 59200, Malaysia
}

Received 3 March 2020; Accepted 25 May 2020

\begin{abstract}
Quantum image watermarking technology is influenced by embedding algorithm, which permanently distorts the embedded quantum carrier image. To protect and authenticate quantum images without damaging the carrier images, this study proposed a quantum image reversible watermarking algorithm combining the most significant qubit (MSQb) and the least significant qubit (LSQb). First, a binary watermark image, a grayscale carrier image, and an empty key image were represented using the novel enhanced quantum representation of digital images. Second, the encrypted qubit of the binary watermark image was matched with the MSQb of the carrier image. If they were equal, then the high qubit of the quantum key image was flipped. Otherwise, the encrypted qubit of the binary watermark image was matched with the XOR value of the lowest two qubits of the carrier image. The LSQb of the carrier image was modified according to the matching result. Finally, the quantum watermarked image was obtained. Simulation experiments and theoretical analysis verified the feasibility and watermark performance of the scheme. Results demonstrate that the scheme achieves correct extraction of quantum image watermark and lossless restoration of original image. The visual quality PSNR of the carrier image embedded with watermark through the scheme is improved by approximately $4 \%$ compared with that through the traditional LSQb method. The study provides a reference for studying and implementing reversible image watermarking technology in the era of quantum computer, which can be applied to harsh copyright protection and authentication of the visual quality of the original carrier image.
\end{abstract}

Keywords: Quantum image processing, Reversible watermarking, Least significant qubit, Most significant qubit

\section{Introduction}

The design of significant quantum algorithm has achieved amazing performance improvement in classical information processing fields [1-2], indicating that quantum computation is a new computing model that can break through the limits of classical computation. With the rapid development of imaging technology and sensor technology, the volume of image data is increasing. Image watermarking technology is also faced with challenging problems, such as computation amount, real-time performance, and algorithm complexity. Therefore, existing studies focus mainly on the application of new quantum computing technology in traditional image processing.

As scholars have carried out intensive study of quantum image processing, the watermarking technology based on quantum image carrier has also been deeply explored. Quantum image information hiding capacity, visual quality and robustness, and the basic function of quantum image watermark embedding and extraction have been realized. However, the original carrier image is permanently distorted [3].

To address this problem, scholars have begun to carry out preliminary studies on reversible data hiding of quantum images using difference expansion technology [4]. Scholars have also proposed the corresponding reversible algorithm to realize lossless restoration of carrier images. However, the algorithm has high complexity and poor watermark performance.

Studying quantum watermarking has become increasingly important. Quantum watermarking can realize the basic function of the watermark and lossless extraction of the original carrier image. The study results are expected to be applied in special image watermarking application scenarios, such as medical image sharing and military image processing, which have theoretical and practical significance. Further optimizing the performance of quantum image watermarking is an urgent matter to ensure the reversibility of algorithm and realize lossless restoration of carrier images.

This study uses novel enhanced quantum representation (NEQR) method [5] to establish a quantum model of the carrier image and watermark image. The latter is encrypted before embedding. The embedding and extraction of watermark and the restoration of the carrier image are analyzed by combining the most significant qubit (MSQb) and the least significant qubit (LSQb) of the quantum carrier image. The study is expected to realize lossless restoration of distorted carrier image while further enhancing the performance of quantum watermarking. In so doing, this study provides reference for optimizing quantum image reversible watermarking technology. 


\section{State of the art}

At present, scholars have studied quantum image processing technology and information hiding technology. Beach [6] first proposed the concept of quantum image processing. Yao [7] studied quantum image processing and its application from two theoretical and experimental aspects. Iliyasu [8] discussed quantum image watermarking and authentication based on restricted geometric transformation and proposed a secure and keyless blind watermarking embedding and recognition scheme for quantum images. Seeing that the quantum circuit of the method was constructed according to watermark image information, only the embedder could extract such an information.

With quantum Hadamard transform as basis, Song [9] proposed a quantum image dynamic watermarking scheme with good visual quality. The scheme belonged to nonblind watermark and needs original carrier data for extraction. $\mathrm{Li}$ [10] used the quantum image representation of logarithmic polar coordinates to embed watermark information into the $\mathrm{LSQb}$ or the second LSQb of the quantum carrier image. The transparency and robustness of the quantum watermark image was enhanced, but the carrier image was distorted permanently. Guo [11] blocked carrier image so that the watermark image could be evenly embedded in each part of the carrier image. The algorithm had good security and robustness, but it overlooked the restoration of the carrier image. Naseri [12] analyzed a secure quantum watermarking scheme using the LSQb and the MSQb. The scheme showed excellent performance in noise attack. The algorithm was not complex but irreversible. Miyake [13] employed the two LSQbs of the carrier image and the watermark image for XOR operation and designed a small-scale quantum circuit of the quantum watermarking scheme. After the watermark was embedded to the carrier image, the two LSQbs were not restored to the original state. Heidari [14-16] studied quantum watermarking technology, proposed quantum watermarking algorithm with multiple keys, and realized copyright protection authentication of quantum data. Like other scholars, he failed to consider the distortion of the carrier image. El-latif [17] embedded the quantum watermark image to the quantum carrier image in the practical scene of telemedicine image sharing. The embedding capacity was improved, but the algorithm did not take reversibility into account, and thus the pixel gray of the carrier image embedded with watermark changes.

Moreover, El-latif [18] presented an image steganography technology based on quantum substitution box, which focused on the embedding capacity of image steganography. In terms of image frequency domain, Wang [19] put forward the LSQb information hiding based on quantum image and explored embedding security. Luo [2021] studied encryption technology for multiple quantum images and quantum color image steganography. All of the aforementioned algorithms led to the distortion of the carrier image and could not restore the original carrier image.

The above studies mainly investigated quantum image information hiding embedding algorithms and algorithm performance and improve watermark performance, such as embedding capacity and visual quality. However, the quantum carrier image is permanently distorted after embedding watermark, thereby limiting the application scope of the quantum watermark. At present, few studies focus on quantum reversible watermarking algorithm and lossless restoration of quantum carrier image on the premise of further optimizing quantum watermarking performance.
In this study, reversible watermarking technology framework of quantum image using NEQR is constructed, and a reversible watermarking algorithm combining the $\mathrm{MSQb}$ and the LSQb is proposed. The performance of the algorithm, such as visual quality and lossless image restoration, is analyzed. The results provide reference for reversible data hiding of quantum images.

The remainder of this study is organized as follows. Section 3 describes quantum image representation model and reversible watermarking framework, analyzes the proposed algorithm, and provides the corresponding quantum implementation circuit. Section 4 verifies and discusses the performance of the algorithm by carrying out simulation experiments and theoretical analysis. The last section draws relevant conclusions.

\section{Methodology}

This section analyzes binary watermark image preprocessing, quantum watermark embedding, extraction algorithm, and carrier image restoration. To intuitively understand the proposed algorithm, the basic framework of the proposed quantum reversible watermarking technology is presented in Fig. 1. Different from previous quantum watermarking methods, quantum reversible watermarking not only realizes the extraction of quantum watermark information but also completes lossless restoration of quantum carrier images. The specific technical details are discussed in the following part.

3.1 Quantum image model and watermark preprocessing NEQR uses two qubit sequences to represent color and location information [5]. This quantum image model can easily realize geometric and color transformation and reconstruct the classical image. The size of the digital image is assumed to be $2^{n} \times 2^{n}$, and the color range is $2^{q}$. Binary bit sequence $C_{Y X}^{q-1} C_{Y X}^{q-2} \ldots C_{Y X}^{1} C_{Y X}^{0}$ is used for coding color value $f(Y, X)$ of position pixel $(Y, X)$, i.e.,

$$
\begin{aligned}
& f(Y, X)=C_{Y X}^{q-1} C_{Y X}^{q-2} \ldots C_{Y X}^{1} C_{Y X}^{0}, C_{Y X}^{k} \in[0,1] \\
& f(Y, X) \in\left[0,2^{q}-1\right]
\end{aligned}
$$

Its NEQR expression is as follows:

$$
\begin{aligned}
|I\rangle & =\frac{1}{2^{n}} \sum_{Y=0}^{2^{n}-1} \sum_{X=0}^{2^{n}-1}|f(Y, X)\rangle \otimes|Y X\rangle \\
& =\frac{1}{2^{n}} \sum_{Y=0}^{2^{n}-1} \sum_{X=0}^{2^{n}-} \bigotimes_{k=0}^{q-1}\left|C_{Y X}^{k}\right\rangle|Y X\rangle
\end{aligned}
$$

In this study, the size of the grayscale carrier image and binary watermark image to be embedded is assumed to be $2^{n} \times 2^{n}$. Using NEQR expression model, the NEQR quantum expression of the grayscale carrier image is as follows:

$$
|C\rangle=\frac{1}{2^{n}} \sum_{Y=0}^{2^{n}-12^{2^{n}-1}} \sum_{X=0}^{7}\left|C_{k=0}^{k}\right\rangle|Y X\rangle
$$

Corresponding to the most significant bit and least significant bit of the classical digital image, the MSQb plane 
of the quantum carrier image in the expression is $\left|C_{Y X}^{7}\right\rangle$, and the LSQb plane is $\left|C_{Y X}^{0}\right\rangle$. Similarly, the NEQR quantum expression of the binary watermark is:

$$
|W\rangle=\frac{1}{2^{n}} \sum_{Y=0}^{2^{n}-12^{2^{n}}-1} \sum_{X=0}\left|W_{Y X}\right\rangle|Y X\rangle
$$

To enhance the security of watermark information, this study uses logistic mapping digital image encryption method to encrypt the binary watermark image embedded in quantum carrier in the following two steps:

Step 1 is choosing suitable parameter $\mu$ and initial value $X_{0}$. When $X_{0} \in[0,1], 3.57 \leq \mu \leq 4$. Use logistic function for $2^{2 n}$ iteration and normalization to generate chaotic sequences $S=\left\{s_{1}, s_{2}, \ldots s_{2^{2 n}}\right\}$.

$X_{\delta+1}=\mu X_{\delta}\left(1-X_{\delta}\right), \delta=0,1, \ldots n$

Step 2 is realizing quantum image XOR operation through chaotic sequence. That is, $2^{2 n}$ quantum controlledNOT (CNOT) gates are used to construct sub-operations

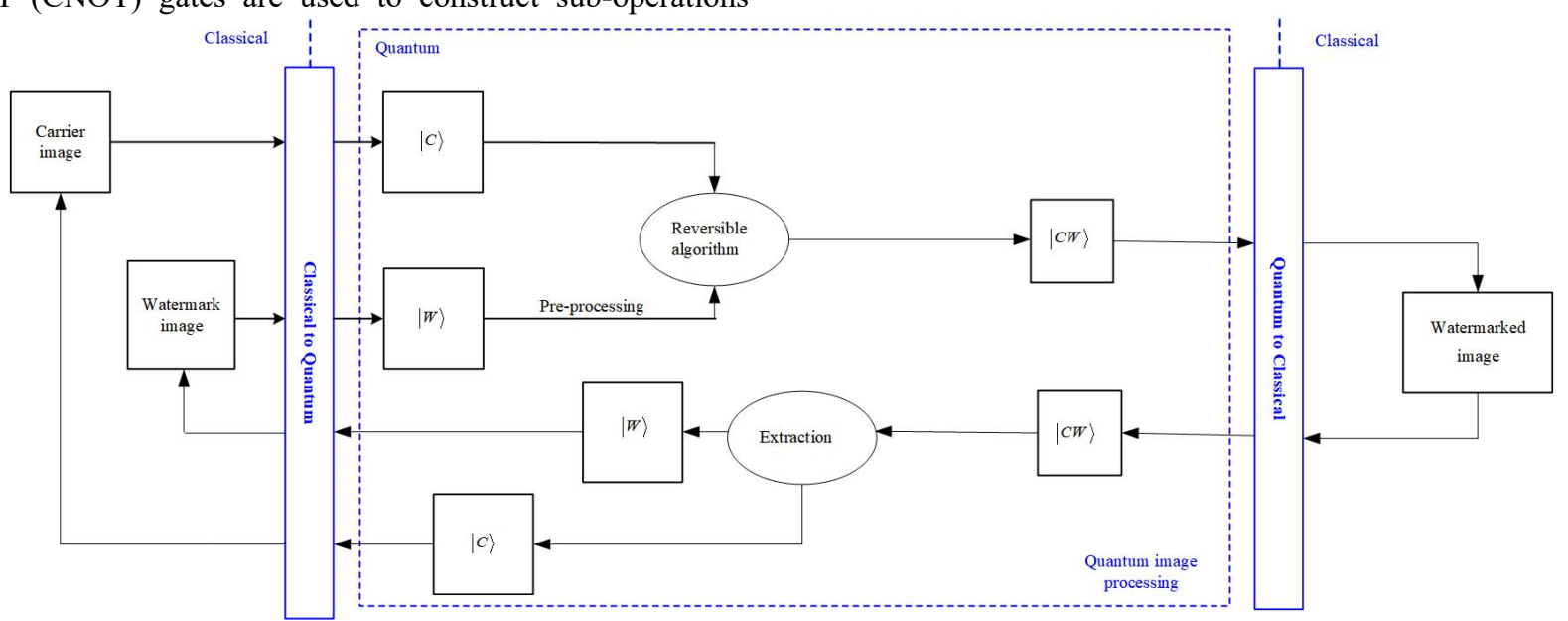

Fig. 1. Basic framework of quantum reversible watermarking

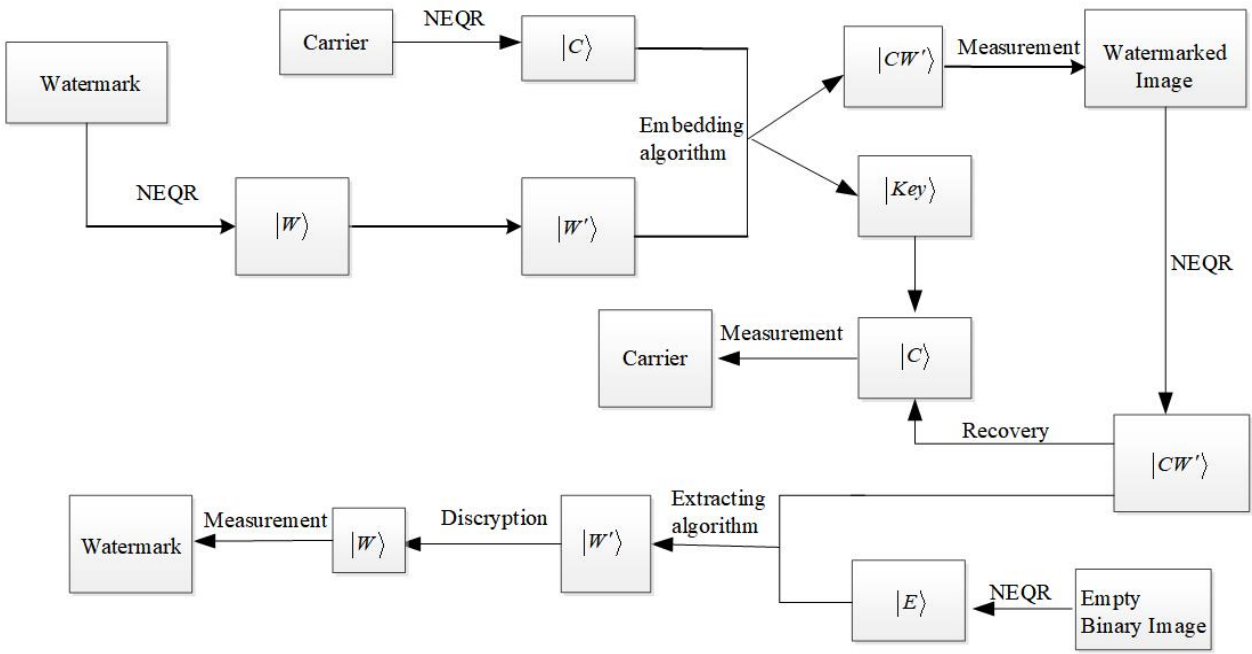

Fig. 2. Embedding and extraction of quantum reversible watermarking 


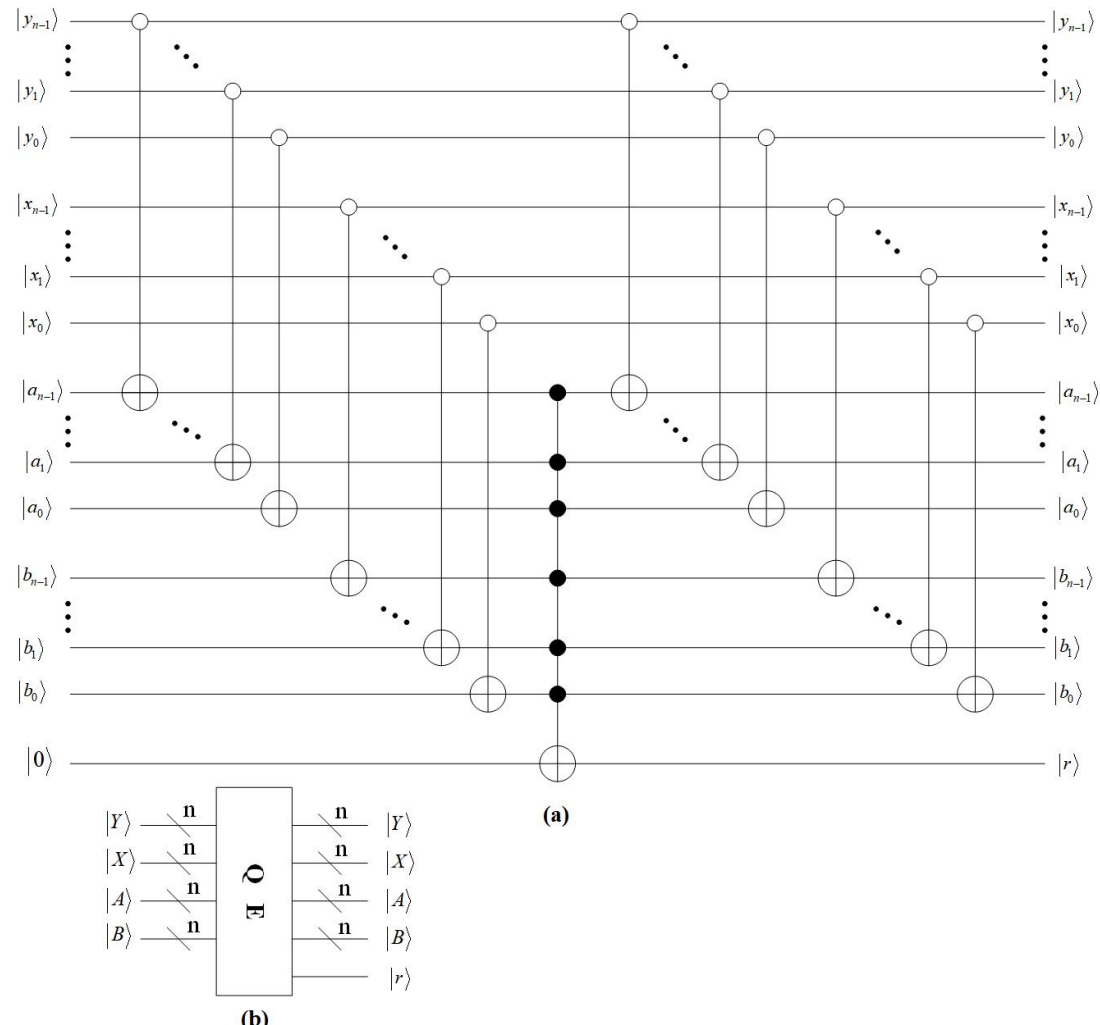

Fig. 3. (a) QE quantum circuit (b) The simplified module

To realize the embedding of quantum watermark, this study uses quantum equation (QE) determination circuit designed by Zhou et al. [22] control the coordinates corresponding to quantum carrier image and watermark image. As shown in Fig. 3, the QE quantum circuit module is employed to compare quantum qubit sequences $|Y\rangle|X\rangle$ and $|A\rangle|B\rangle$, where $|Y\rangle=\left|y_{n-1} \ldots y_{1} y_{0}\right\rangle$, $|X\rangle=\left|x_{n-1} \ldots x_{1} x_{0}\right\rangle,|A\rangle=\left|a_{n-1} \ldots a_{1} a_{0}\right\rangle,|B\rangle=\left|b_{n-1} \ldots b_{1} b_{0}\right\rangle$. The comparison result is expressed by output qubit $|r\rangle$. If $|r\rangle=|1\rangle$, then $|Y X\rangle=|A B\rangle$; otherwise, $|Y X\rangle \neq|A B\rangle$.

In this algorithm, the coordinate qubit sequences of three quantum images of the same size, namely, quantum carrier image $|C\rangle$, encrypted watermark image $\left|W^{\prime}\right\rangle$, and the empty quantum key image $|k e y\rangle$ are compared using $\mathrm{QE}$ circuit. When the output qubits satisfy $\left|r_{1}\right\rangle=\left|r_{2}\right\rangle=|1\rangle$, the coordinates of the three quantum images with the same size correspond to one another.

(1) An empty quantum key image is prepared, and the qubit value of each pixel is initialized to $|00\rangle$. The NEQR expression is as follows:

$$
|K e y\rangle=\frac{1}{2^{n}} \sum_{Y=0}^{2^{n}-12^{2^{n}-1}} \sum_{X=0}^{1}\left|\operatorname{Key}_{k=0}^{k}\right\rangle|Y X\rangle
$$

(2) The MSQb plane $\left|C_{Y X}^{7}\right\rangle$ of quantum carrier images and the binary watermark image $\left|W_{Y X}^{\prime}\right\rangle$ to be embedded are matched. If $\left|C_{Y X}^{7}\right\rangle=\left|W_{Y X}^{\prime}\right\rangle$, then the high qubit of the quantum key image is $|1\rangle$, that is, $\left|\operatorname{Key}_{Y X}^{1}\right\rangle=|1\rangle$; otherwise, $\left|K e y_{Y X}^{1}\right\rangle=|0\rangle$.

(3) When $\left|C_{Y X}^{7}\right\rangle \neq\left|W_{Y X}^{\prime}\right\rangle$, the two LSQb planes of the quantum carrier image are operated by CNOT, namely, $\left|C_{Y X}^{1}\right\rangle \oplus\left|C_{Y X}^{0}\right\rangle$.

(4) The above XOR value and the binary watermark image qubit $\left|W_{Y X}^{\prime}\right\rangle$ are matched. If $\left|C_{Y X}^{1}\right\rangle \oplus\left|C_{Y X}^{0}\right\rangle=\left|W_{Y X}^{\prime}\right\rangle$, then the low qubit of the quantum key image is $|1\rangle$, i.e., $\left|\operatorname{Key}_{Y X}^{0}\right\rangle=|1\rangle$; otherwise, $\left|\operatorname{Key}_{Y X}^{0}\right\rangle=|0\rangle$.

(5) When $\left|K e y_{Y X}^{0}\right\rangle=|0\rangle$, the LSQb of quantum carrier image is reversed, i.e., $\left|C_{Y X}^{0}\right\rangle=\left|\overline{C_{Y X}^{0}}\right\rangle$. The XOR value of the LSQb and the second LSQb are matched with the watermark image qubit, i.e., $\left|C_{Y X}^{1}\right\rangle \oplus\left|\overline{C_{Y X}^{0}}\right\rangle=\left|W_{Y X}^{\prime}\right\rangle$

(5) After embedding, the key image $|K e y\rangle$ is generated. The quantum key image is used for subsequent extraction of quantum watermark and restoration of original quantum carrier image. Meanwhile, the steganographic image $\left|C W^{\prime}\right\rangle$ after embedding watermark is obtained. The expression is as follows:

$$
\left|C W^{\prime}\right\rangle=\frac{1}{2^{n}} \sum_{Y=0}^{2^{n}-1} \sum_{X=0}^{2^{n}-1} \bigotimes_{k=0}^{7}\left|C W_{Y X}^{\prime k}\right\rangle|Y X\rangle
$$

The quantum multi-CNOT and the above QE quantum circuit are used. The reversible logic quantum circuit of the embedding algorithm is shown in Fig. 4.

\subsection{Quantum watermark extraction}


Generally speaking, quantum watermark extraction is the inverse process of quantum watermark embedding, and it involves the following steps:

(1) Prepare an empty quantum binary image $|E\rangle$, and the NEQR expression is:

$$
|E\rangle=\frac{1}{2^{n}} \sum_{Y=0}^{2^{n}-1} \sum_{X=0}^{2^{n}-1}\left|E_{Y X}\right\rangle|Y X\rangle
$$

All values of $\left|E_{Y X}\right\rangle$ are initialized to $|0\rangle$.

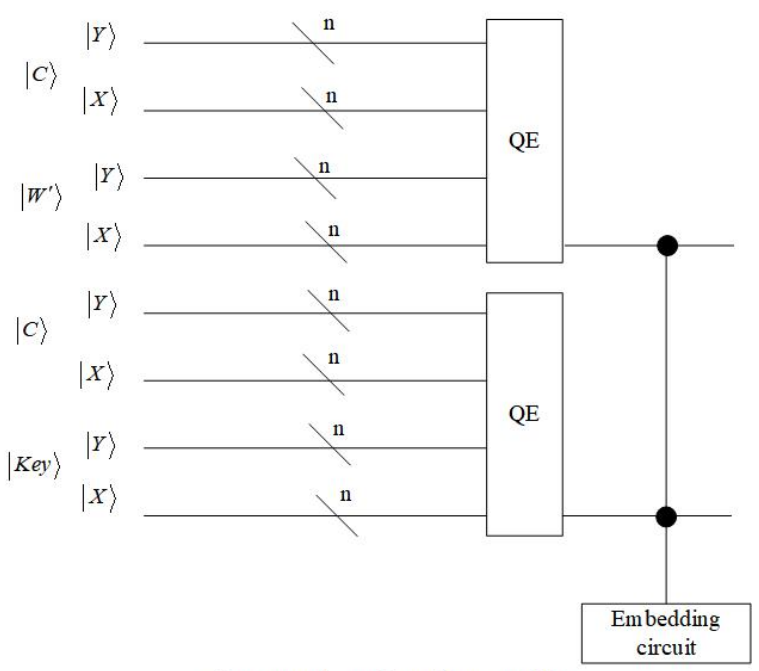

(a) Comparison of coordinate module

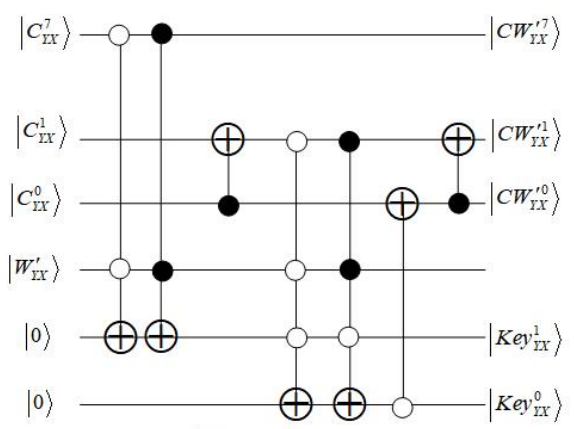

(b) Embedding circuit

Fig. 4. Comparison of coordinate quantum module and embedding circuit

(2) According to the above embedding process, if the high qubit $\left|K e y_{Y X}^{1}\right\rangle$ of the key image is $|1\rangle$, then the value of the MSQb of the steganographic image is the value of the watermark image qubit, i.e., $\left|E_{Y X}\right\rangle=\left|C W_{Y X}^{\prime 7}\right\rangle$.

(3) If the high qubit $\left|\operatorname{Key}_{Y X}^{1}\right\rangle$ of the key image is $|0\rangle$, then the value is determined according to the value of qubit $\left|K e y_{Y X}^{0}\right\rangle$. When $\left|K e y_{Y X}^{0}\right\rangle=|1\rangle,\left|E_{Y X}\right\rangle=\left|C W_{Y X}^{\prime 1}\right\rangle \oplus\left|C W_{Y X}^{\prime 0}\right\rangle$; otherwise, $\left|E_{Y X}\right\rangle=\left|C W_{Y X}^{\prime 1}\right\rangle \oplus\left|\overline{C W_{Y X}^{\prime 0}}\right\rangle$.

(4) The original quantum watermark $|W\rangle$ is obtained by decrypting the extracted quantum watermark image. The quantum extraction circuit is shown in Fig. 5.

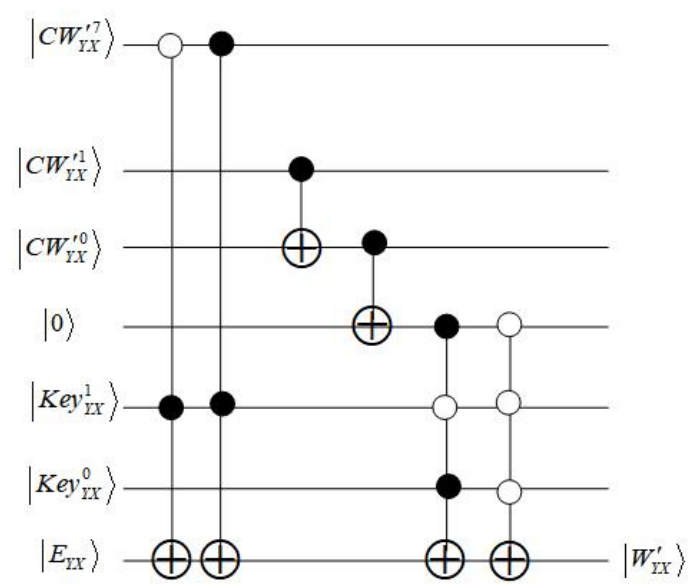

Fig. 5. Extraction of quantum watermark

\subsection{Restoration of quantum carrier image}

Permanent distortion of the carrier image is inevitable in watermark embedding, and the proposed algorithm is no exception. However, from the above analysis of embedding and extraction, the algorithm can restore the steganographic image after embedding watermark and get the lossless original quantum carrier image. The specific steps are as follows:

(1) When the key image satisfies $\left|K e y_{Y X}^{0}\right\rangle=|0\rangle$, the LSQb $\left|C_{Y X}^{0}\right\rangle$ of the carrier image is reversed during embedding. According to the key image, the LSQb of the steganographic image must be reversed after embedding watermark, i.e., $\left|C_{Y X}^{0}\right\rangle=\left|\overline{C W_{Y X}^{\prime 0}}\right\rangle$, so that the LSQb value of the original carrier image can be obtained.

(2) In addition, all qubit planes of the quantum carrier image are modified in the situations corresponding to the key image qubit.

When the restoration of the original quantum carrier image is controlled by the key, CNOT of the embedded watermark image can restore the original carrier image, and the proposed algorithm is simple and effective.

\section{Result Analysis and Discussion}

Limited by universal quantum computers, the proposed scheme and its quantum circuit are simulated by a notebook computer with MATLAB 2014b. Its hardware is Intel (R) Core (TM) i5-7200U CPU 2.70-GHz 8.00-GB RAM.

In the simulation experiment, two gray images with the size of $256 \times 256$ ("Lena" and "Cameraman") as quantum carrier images and binary images with the size of $256 \times 256$ ("Airplane" and "Peppers") are taken as the quantum watermark image to be embedded. The test images are shown in Fig. 6. The binary watermark image Peppers was conducted with logistic chaos encryption and embedded into the carrier image Lena and Cameraman. The visual effects of the original carrier image, the carrier image embedded with watermark (steganographic image), and the restored carrier image are shown in Fig. 7. From the perspective of human visual system, the visual quality of the image embedded with watermark has high fidelity, and the desired effect is achieved. 


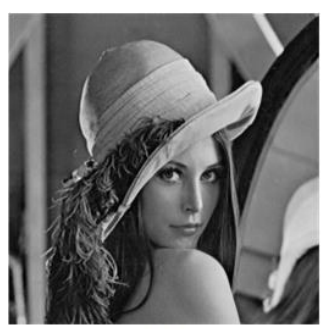

(a)

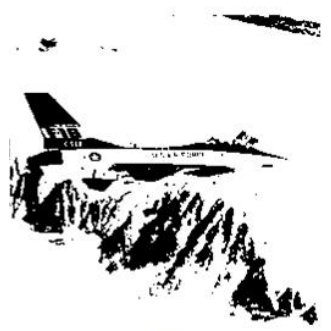

(c)

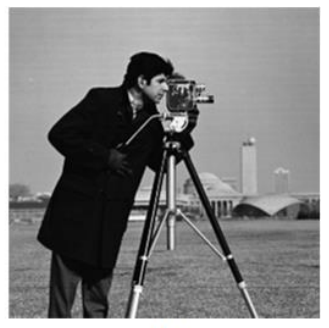

(b)

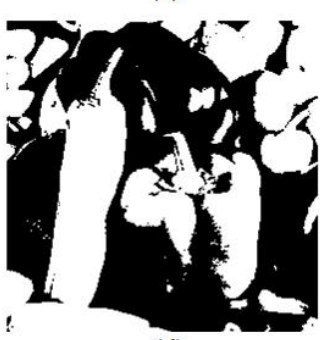

(d)
Fig. 6. Test images: (a) and (b) are grayscale carrier images, (c) and (d) are binary watermark images

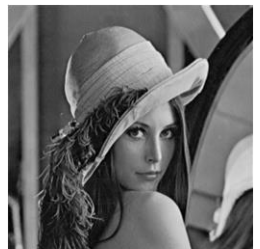

(a)

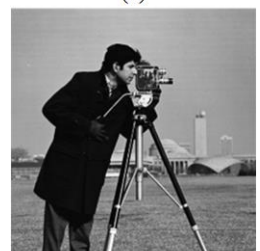

(d)

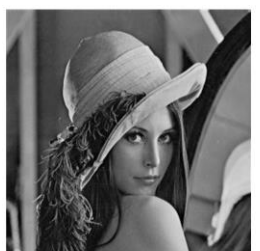

(b)

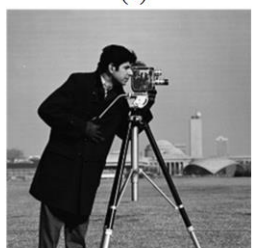

(e)

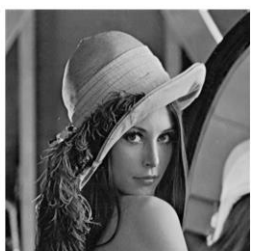

(c)

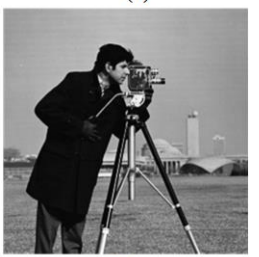

(f)
Fig. 7. Comparison of visual effects: (a) and (d) are original carrier images, (b) and (e) are steganographic images embedded with watermark, (c) and (f) are restored carrier images

\subsection{Peak signal to noise ratio (PSNR)}

PSNR is an important index when judging the similarity of two images (carrier image embedded with watermark and original carrier image). The larger the value, the higher the similarity of the two images. For carrier image $I$ with the size of $m \times n$ and the corresponding carrier image $J$ embedded with watermark, PSNR is defined as follows:

$$
P S N R=10 \log _{10} \frac{M A X_{I}^{2}}{M S E}=20 \log \frac{M A X_{I}}{\sqrt{M S E}}
$$

where $M A X_{I}$ represents the maximum gray value of the image color. MSE is the mean squared error, which is defined as

$$
M S E=\frac{1}{m n} \sum_{i=0}^{m-1} \sum_{j=0}^{n-1}[I(i, j)-I(i, j)]^{2}
$$

After embedding the binary watermark image into the carrier image, the corresponding PSNR values are obtained (Table 1). The values of PSNR are higher than $60 \mathrm{~dB}$. Compared with that of the simple least significant bit substitution method, the PSNR value of the proposed method is increased by approximately $3 \%$. Compared with the enhanced watermarking scheme using the LSQb method [23], the embedding capacity of the proposed algorithm is one bit per pixel, which is much higher than that of the enhanced watermarking scheme, but its PSNR value is equivalent to that of the proposed method. Therefore, the PSNR value further verifies the ideal embedding visual quality of the scheme. In addition, the PSNR values of the restored carrier image are calculated, and all values are Inf. The algorithm achieves reversible data hiding, and lossless restoration is realized for the carrier image embedded with watermark.

Table 1. The corresponding PSNR values

\begin{tabular}{c|c|c|c}
\hline Carrier/watermark & $\begin{array}{c}\text { Simple } \\
\text { LSQb } \\
\text { substitution }\end{array}$ & $\begin{array}{c}\text { Scheme } \\
\text { in Ref. } \\
{[\mathbf{2 3}]}\end{array}$ & $\begin{array}{c}\text { Proposed } \\
\text { scheme }\end{array}$ \\
\hline Lena/Peppers & 58.4895 & 61.2322 & 61.4948 \\
Cameraman/Peppers & 58.5065 & 61.3509 & 61.3265 \\
Lena/Airplane & 58.1260 & 60.9854 & 61.1560 \\
Cameraman/Airplane & 58.3402 & 60.8081 & 61.2635 \\
\hline
\end{tabular}

\subsection{Analysis of computational complexity}

The complex unitary operation of multiple qubits can be decomposed into single qubit logic gate and two qubit CNOT gates [24]. Therefore, the complexity of quantum computation is dependent on the number of basic quantum logic gates. On this basis, we choose the CNOT gate as the basic logic unit. The complexity of the proposed algorithm is analyzed as follows.

As shown in Fig. 4 (a), the coordinate consequences of two $2 n$ qubits are compared using quantum equivalent circuits. According to the quantum equivalent circuit, the module is composed of $4 n$ CNOT gates and a $2 n$-CNOT gate. The complexity of the multi-controlled quantum bit $n$-CNOT is nearly $12 n-9$. Therefore, the complexity of the two quantum $\mathrm{QE}$ circuits is $2 \times(4 n+12 \times 2 n-9)(n \geq 3)$. As shown in Fig. 4 (b), two qubits $\left|r_{1}\right\rangle$ and $\left|r_{2}\right\rangle$ are output through quantum equivalent circuit. The embedded circuit consists of $23-\mathrm{CNOT}, 2$ 4-CNOT, and 2 5-CNOT, and the complexity is $2 \times(12 \times 4-9+12 \times 3-9+12 \times 5-9)$. The complexity of reversible watermarking algorithm embedding is only $O(n)$. As shown in Fig. 5, the coordinate is controlled using QE circuit in watermark extraction. The extraction is the inverse process of embedding with simple CNOT operation, which achieves lossless extraction of the original carrier image with the same complexity. If the complexity of quantum image preparation and equivalent quantum computation is neglected, then the total complexity of the algorithm is $O(n)$. Compared with that of the classical reversible watermarking method of least significant bit substitution, the complexity of the proposed scheme is approximately $O\left(2^{2 n}\right)$ because all pixels are processed. This algorithm makes the utmost of the entanglement and superposition characteristics of quantum computation and obviously enhances the efficiency of image processing.

\section{Conclusion}

Watermark embedding often leads to the permanent distortion of the carrier image. To realize lossless restoration of the original carrier image, this study uses NEQR quantum image model and combines the LSQb and the MSQb to 
analyze the embedding and extraction of quantum watermark and the restoration of carrier image. Finally, the following conclusions are drawn:

(1) Quantum watermark embedding algorithm is reversible and can realize lossless restoration of the original carrier image.

(2) The quantum circuit of the proposed scheme is designed using a series of quantum gate operation. The circuit is simple, and the computational complexity is $O(n)$.

(3) The steganographic image embedded with quantum watermark has high visual quality, which can realize blind extraction of quantum watermark image without referring to the original image.

Based on the NEQR quantum image model, this study proposes a quantum reversible watermarking algorithm integrating the LSQb and the MSQb. The performance of quantum watermarking and lossless restoration of quantum carrier images are analyzed and discussed. The results provide a reference for the study of quantum reversible watermarking. Limited by steganographic capacity, the algorithm can be further optimized to meet the requirements of large-capacity reversible watermarking technology.

\section{Acknowledgements}

This work was supported by Hunan Provincial Natural Science Foundation of China (Grant No. 2020JJ40509) and the Research Foundation of Education Bureau of Hunan Province, China (Grant Nos. 18B420 and 19B512).

This is an Open Access article distributed under the terms of the Creative Commons Attribution License

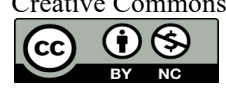

\section{References}

1. P. W. Shor, "Algorithms for quantum computation: discrete logarithms and factoring". In: Proceedings of 35th Annual Symposium on Foundations of Computer Science, Santa Fe, NM, USA: IEEE, 1994, pp. 124-134.

2. L. K. Grover, "A fast quantum mechanical algorithm for database search". In: Proceedings of the 28th Annual ACM symposium on the Theory of Computing, New York, USA: ACM, 1996, pp. 212219.

3. Z. Qu, Z. Cheng, W. Liu, and X. Wang, "A novel quantum image steganography algorithm based on exploiting modification direction". Multimedia Tools and Applications, 78(7), 2019, pp.7981-8001.

4. S. Xiang, H. Li, and T. Song, "Reversible data hiding algorithm in NEQR quantum images (in Chinese)". Journal of Cyber Security, 3(6), 2018, pp. 78-91.

5. Y. Zhang, K. Lu, Y. Gao, and M. Wang, "NEQR: a novel enhanced quantum representation of digital images". Quantum Information Processing, 12(8), 2013, pp.2833-2860.

6. G. Beach, C. Lomont, and C. Cohen, "Quantum image processing (QuIP)". In: Proceedings of the 32nd Applied Imagery Pattern Recognition Workshop, Washington, USA: IEEE, 2003, pp.39-44.

7. X. W. Yao, H. Wang, Z. Liang et al., "Quantum image processing and its application to edge detection: theory and experiment". Physical Review X, 7(3), 2017, pp.031041.

8. A. M. Iliyasu, P. Q. Le, F. Dong, and K. Hirota, "Watermarking and authentication of quantum images based on restricted geometric transformations". Information Sciences, 186(1), 2012, pp. 126-149.

9. X. Song, S. Wang, A. A. Abd El-Latif, and X. Niu, "Dynamic watermarking scheme for quantum images based on Hadamard transform". Multimedia Systems, 20(4), 2014, pp. 379-388.

10. T. Li, Z. Cheng and Z. Qu, "Robust quantum watermarking algorithm based on key to implement controlled Least significant qubit modification technique (in Chinese)". Application Research of Computers, 36(4), 2019, pp.1133-1138.

11. H. Guo, Y. Du and Q. XU, "Quantum image watermarking algorithm based on blocked spatial domain (in Chinese)". Chinese Journal of Quantum Electronics, 35(5), 2018, pp. 527-532

12. M. Naseri, S. Heidari, M. baghfalaki et al., "A new secure quantum watermarking scheme" Optik, 139, 2017, pp.77-86.
13. S. Miyake and K. Nakamae, "A quantum watermarking scheme using simple and small-scale quantum circuits". Quantum Information Processing, 15(5), 2016, pp.1849-1864.

14. S. Heidari, R. Gheibi, M. Houshmand, and K. Nagata, "A robust blind quantum copyright protection for colored images based on owner's signature". International Journal of Theoretical Physics, 56(8), 2017, pp.2562-2578.

15. S. Heidari and E. Farzadnia, "A novel quantum LSB-based steganography method using the Gray code for colored quantum images". Quantum Information Processing, 16(10), 2017, pp.242.

16. S. Heidari, M. R. Pourarian, R. Gheibi, M. Naseri, and M. Houshmand, "Quantum red-green-blue image steganography". International Journal of Quantum Information, 15(5), 2017, pp.1750039.

17. A. A. A. El-latif, B. Abd-el-atty, and M. S. Hossain, "Efficient quantum information hiding for remote medical image sharing". IEEE Access, 6, 2018, pp.21075-21083.

18. A. A. A. El-latif, B. Abd-el-atty, and S. E. Venegas-andraca, "A novel image steganography technique based on quantum substitution boxes". Optics and Laser Technology, 116, 2019, pp. 92-102.

19. S. Wang, J. Sang, X. Song, and X. Niu, "Least significant qubit (LSQb) information hiding algorithm for quantum image". Measurement, 73, 2015, pp.352-359.

20. G. Luo, R.-G. Zhou, and W.-W. Hu, "Novel quantum secret image sharing scheme". Chinese Physics B, 28(4), 2019, pp. 040302.

21. G. Luo, L. Shi, Ammar. Oad et al. "Steganography of quantum color images based on blocking and gray Level difference". Journal of Engineering Science and Technology Review, 13(1), 2020, pp. 98-105.

22. R-G. Zhou, W. Hu, G. Luo et al. "Quantum realization of the nearest neighbor value interpolation method for INEQR". Quantun Information Processing, 17(7), 2017, pp.166.

23. G. Luo, R-G. Zhou, W. Hu et al. "Enhanced Least significant qubit watermarking scheme for quantum images". Quantun Information Processing, 17(11), 2018, pp.299.

24. M. A. Nielsen and I. L. Chuang, "Quantum computation and quantum information". Cambridge: Cambridge University Press, The UK, 2000, pp.3-35. 